\title{
Sexuelle und geschlechtliche Vielfalt in der Altenpflege
}

\author{
Diversity-sensibel pflegen Nicht-heterosexuelle Personen fühlen sich mit \\ ihrer sexuellen und geschlechtlichen Identität in traditionellen Einrichtungen \\ der Altenpflege oftmals nicht willkommen. Bisher gibt es auch wenig Erkennt- \\ nisse zur ihrer Situation. Ergebnisse einer Studie an der Alice Salomon Hoch- \\ schule Berlin werden hier nun vorgestellt.
}

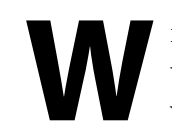
ieso denn jetzt LSBTI? Wir pflegen jeden gleich. Dies wurde oft geäußert bei der Suche nach Interviewpartner*innen auf einem Heimleiter ${ }^{\star}$ innen-Treffen im Rahmen des Forschungsprojekts Gleichgeschlechtliche Lebensweisen und Pflege im Alter (GLEPA). Das deutet darauf hin, wie

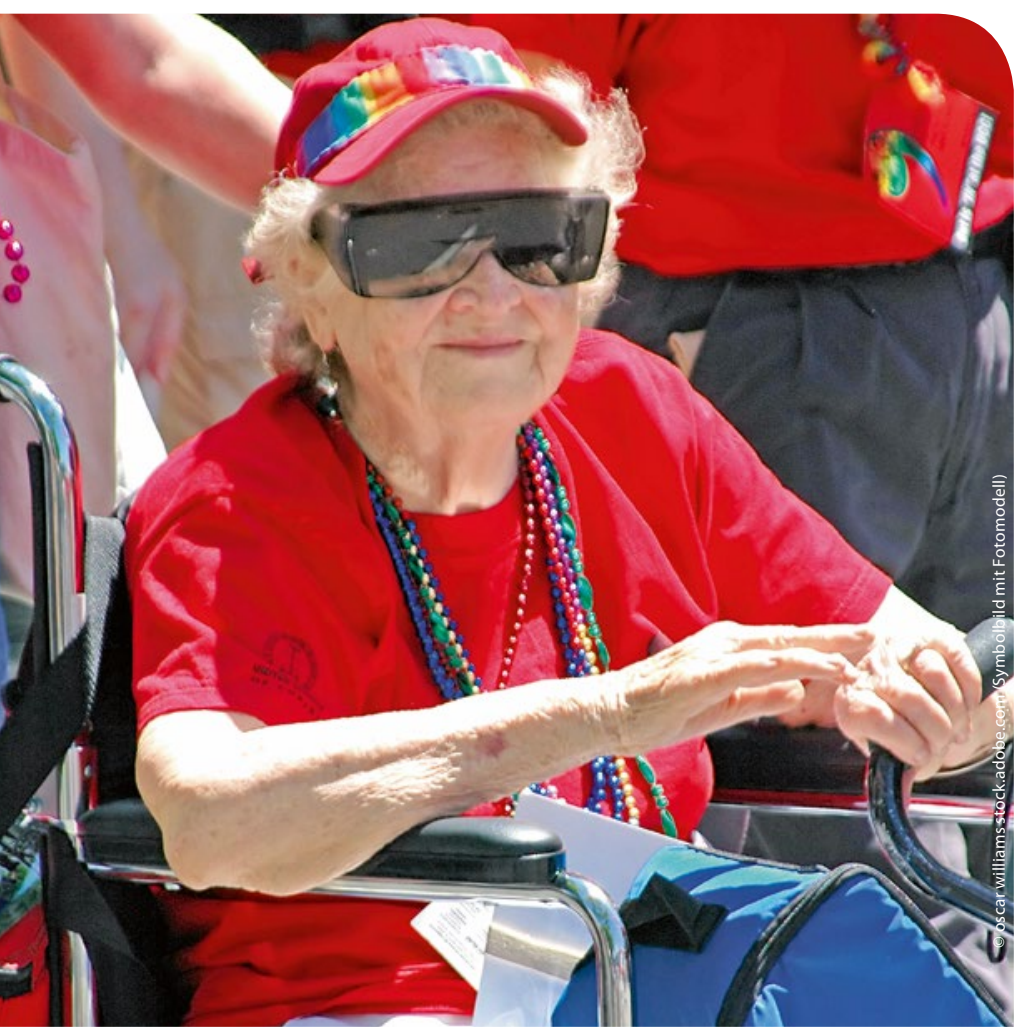

LSBTI-Pflegebedürftige fühlen sich in traditionellen Einrichtungen der Altenpflege oft nicht willkommen und wollen sich häufig auch nicht erklären.
Pflege mit ihren Rahmenbedingungen wahrgenommen wird, außerdem auf das Übersehen von Minoritäten im Pflegealltag. Der Beitrag thematisiert die soziodemografische Situation und Bedarfe von LSBTI $^{*}$-Pflegebedürftigen, womit lesbische, schwule und bisexuelle sowie trans ${ }^{\star}$ und inter ${ }^{\star}$ Senior ${ }^{\star}$ innen gemeint sind. Die GLEPA-Studie wurde von 2015 bis 2017 an der Alice Salomon Hochschule Berlin in Kooperation mit der Hochschule für Wirtschaft und Recht Berlin durchgeführt. Hierfür wurden biografisch-narrative Interviews mit LSBTI*-Pflegebedürftigen und Expert*inneninterviews aus der Heim- und Wohnbereichsleitung geführt.

\section{Stand der Forschung}

Die Bevölkerungsgruppe, die mit dem Akronym LSBTI^ beschrieben wird, ist eine höchst heterogene Gruppe, die in der Gerontologie und den Pflegewissenschaften bislang nur wenig Beachtung fand. Neuere Studien zu LSBTI*, Alter(n) und Pflege zeigen, dass LSBTI $^{*}$-Personen sich mit ihrer sexuellen und geschlechtlichen Identität in „traditionellen“ Einrichtungen der Altenpflege oftmals nicht willkommen fühlen, Unsichtbarkeiten beschreiben und sich im Alter nicht mehr (proaktiv) erklären können oder wollen. Qualitative Studien beschreiben beispielsweise die Befürchtung von LSB-Senior*innen, dass sie im Alter ihr offenes Leben als LSBSenior ${ }^{\star}$ in nicht so weiterführen können, wie sie es sich über Jahre erkämpft hatten. Meist negative Folgen sogenannter heteronormativer Strukturen in der Altenhilfe wurden in angloamerikanischen Studien und insbesondere für den pflegerischen Bereich auch in deutschen Studien nachgewiesen (Literatur im HEILBERUFE eMag).

\section{Unterstützung im Alter und in der Pflege}

Wird bei heterosexuellen Senior*innen für die soziale und pflegerische Unterstützung im Alter meist auf Hilfe von Kindern, Ehepartnern und anderen Verwandten gesetzt, so ist dies bei LSBTI*Senior ${ }^{\star}$ innen nicht unbedingt der Fall. Bei den älteren Homosexuellen sind in der Regel Freund*innen auf Platz eins der Unterstützungshierarchie im Alter. Bei LSBT*-Senior*innen können Freund- 
schaftsnetzwerke, die als sogenannte Wahlfamilie verstanden werden, als Rückgrat der sozialen Unterstützung angesehen werden. Für Bisexuelle, trans ${ }^{\star}$ und inter ${ }^{\star}$ liegen hierzu keine valide Daten vor. Auch für „nicht-heterosexuelle“ Senior*innen gelten Partner*innen als Hauptunterstützungsressource für die pflegerische Versorgung. Doch ist darauf hinzuweisen, dass insbesondere bei schwulen älteren Männern von einem hohen Anteil an Singles auszugehen ist. Haben heterosexuelle Frauen im Alter von $50+\mathrm{zu}$ circa $85 \%$ mindestens ein Kind, so sind LSBT*-Senior*innen häufig kinderlos. In einer Studie zu älteren lesbischen Frauen gaben knapp 15\% der befragten Lesben zwischen 50 und 73 Jahren an, ein Kind oder mehr zu haben (etwas darunter liegen die Werte für schwule Senioren).

\section{Verantwortung der Altenhilfe und Altenpflege}

Über den Lebensverlauf haben viele LSBTI*-Senior*innen Minderheitenstress erlebt: Angehörige z.B. sexueller Minderheiten erfahren überproportional Diskriminierung und Stigmatisierung aufgrund ihrer sexuellen oder geschlechtlichen Identität mit unterschiedlichen Folgen für ihre psychische Gesundheit.

Daraus resultierend bestehen bei LSBTI*-Senior*innen vielfältige herausfordernde Situationen in der Lebensphase Alter und insbesondere bei eintretender Pflegebedürftigkeit (z.B. HIV/Aids bei schwulen Männern). Die Kriminalisierung von Homosexualität durch den Strafrechtsparagraphen 175 hinterlässt in Biografien insbesondere von schwulen Männern Spuren. Folgen dieser Repression zeigen sich auch im pflegerischen Handeln.

\section{Gefahr der Re-Traumatisierung}

Insbesondere bei trans ${ }^{\star}$ und intergeschlechtlichen Senior ${ }^{\star}$ innen besteht zudem die Gefahr von Re-Traumatisierungen durch häufig negative Erfahrungen mit dem Gesundheitssystem in früheren Lebensphasen. Diese Risiken für eine gute Lebens- und Pflegequalität im Alter sind vor dem Hintergrund der Angaben zu Partnerschaften, Kinderlosigkeit und Alleinleben zu betrachten. Diese soziodemografischen Kennzeichen begründen eine besondere Abhängigkeit von formellen Pflegeleistungen, weshalb den Regeldiensten der Altenhilfe für LSBT ${ }^{*}$-Senior ${ }^{\star}$ innen eine besondere Verantwortung zukommt. Das gilt besonders auch für das Fachpersonal in der Altenhilfe bzw. in der ambulanten und stationären Pflege - bis hin zur Sterbebegleitung. Aufgrund der häufig intimen und persönlichen Hilfestellungen und der Abhängigkeit durch Dritte wird bei LSBTI*-Senior ${ }^{*}$ innen ein Bedarf nach Personal mit biografischen und lebensweltlichen Kompetenzen im Bereich LSBTI $^{\star}$ festgestellt, der auch für andere Bevölkerungsminderheiten beobachtet wird.

Doch zeigt sich in Studien gegenüber Altenhilfeeinrichtungen ein starkes Unbehagen seitens von Homosexuellen, das u.a. aufgrund der über den Lebenslauf gemachten Erfahrungen mit dem Gesundheitssystem gilt. Sowohl schwule Männer als auch lesbische Frauen bewerten Einrichtungen der Altenhilfe hinsichtlich einer Berücksichtigung von LSB-Senior*innen negativ (je nach Einrichtungsart zwischen $50 \%$ und $81 \%$ Ablehnung bei schwulen Senioren, $96 \%$ bei lesbischen Seniorinnen). Die Perspektive von trans* und intergeschlechtlichen Senior ${ }^{\star}$ innen bleibt in diesen Studien unberücksichtigt.

\section{+ WICHTIGE BEGRIFFE}

\begin{abstract}
LSBTI*: steht für lesbisch (L), schwul (S) oder bisexuell (B) und für trans* $(T)$ und intergeschlechtliche (I) Menschen. Das Sternchen ${ }^{*}$ ) soll auch weitere, bisher nicht genannte, sexuelle und geschlechtliche Identitäten und Selbstbeschreibungen mit einbeziehen und auf diese hinweisen.
\end{abstract}

Sexuelle Vielfalt: umfasst die sexuellen Identitätskategorien lesbisch, schwul und bisexuell. Lesbisch, schwul und bisexuell sind Selbstbeschreibung der eigenen sexuellen Orientierung bzw. Identität.

Geschlechtliche Vielfalt: umfasst die Begriffe trans* und inter*, die sich der Logik eines Zwei-GeschlechterSystems entziehen. Mit Geschlechtsidentität wird das Zugehörigkeitsgefühl zu einem Geschlecht beschrieben.

trans*: so bezeichnen sich Menschen, wenn deren Geschlechtsidentität von dem Geschlecht abweicht, das ihnen bei der Geburt zugewiesen wurde. Trans* ist ebenso ein Oberbegriff für verschiedene trans*-Identitäten und Lebensweisen (transgender, transident, transsexuell).

Inter*: steht für intergeschlechtliche oder intersexuelle Menschen, die mit Variationen der Geschlechtsmerkmale geboren werden. Inter* betrifft also die Beschaffenheit des jeweiligen Körpers.

\section{Rahmenbedingungen verstärken „Unsichtbarmachen“}

Die GLEPA-Studie beschreibt anhand der empirischen Analyse mit LSBTI*-Pflegebedürftigen eine sogenannte „Heteronormativität“, die sich auf die Praxis in der Altenhilfe auswirkt. Heteronormativität meint ein generelles Unterstellen einer Heterosexualität bei (neuen) Klient ${ }^{*}$ innen und zu Beratenden in der Altenhilfe und in der Pflege. In der ambulanten wie stationären Altenpflege wird dieses „Unsichtbarmachen“ von Menschen mit ihren Lebensgeschichten durch die in der Pflege schwierigen Rahmenbedingungen verstärkt.

Die alltägliche Arbeit in der Pflege ist durch einen Mangel an Zeit und Fachpersonal gekennzeichnet, was die in der Pflege Tätigen vor Herausforderungen stellt. Das Ziel einer personenzentrierten Pflege kann so generell kaum erreicht werden. Interviews mit Heimleiter*innen im Rahmen von GLEPA offenbaren, dass Maßnahmen des Diversity Management in der Altenpflege fehlen und bei einer Sensibilisierung für Bedarfe und der Vermeidung von Diskriminierung helfen würden.

\section{Blick auf Biographien und Vulnerabilitäten unerlässlich}

Sensibilisierungen über Fort- und Weiterbildung zielen auf eigene Ängste, mögliche Vorurteile, Wissen um mögliche heteronormative Strukturen im Betrieb sowie um Diskriminierungserfahrungen bei den älteren und hochaltrigen LSBTI $^{*}$-Pflegebedürftigen, aber 


\section{PFLEGE ALLTAG}

auch Ideen zur Angehörigenarbeit und Lebensweltkenntnissen (Stichwort: Wahlfamilie). Die Autor ${ }^{*}$ innen der Studie verweisen insbesondere auf Ressourcen, die Mitarbeiter*innen, die selbst offen $\mathrm{LSBT}^{\star}$ sind, für die Pflege einbringen können in Hinsicht auf das Erkennen von LSBTI*-Pflegebedürftigen sowie bei Lebensweltkenntnissen für eine gute soziale Teilhabe. Diese Mitarbeitenden beschrieben in den Interviews LSBT*-Personen differenzierterer und weniger stereotypenhaft wie ihre heterosexuellen Kolleg*innen. Handlungsempfehlungen für eine diversity-sensible Pflege sind eng verknüpft mit diesen Kenntnissen und einer Willkommenskultur - beides wird in einigen Pflegeeinrichtungen insbesondere in den Niederlanden und in deutschen Metropolen über Diversity-Zertifikate bereits verfolgt („Pink Passkey“, „Qualitätssiegel Lebensort Vielfalt"). Ein neues bundesweites Modellprojekt vom AWO Bundesverband e.V. zur Öffnung der Altenhilfe für LSBTI ${ }^{*}$ wird in 2021 ein Handbuch mit Konzeption und Trainingsmaterialien an die deutschen Sozialverbände zu diesem Thema abgeben.

Bei dem Thema LSBTI*, Alter(n) und Pflege wird deutlich, dass für die offene und stationäre Altenhilfe die Soziale Arbeit besondere Bedeutung erlangt (beispielsweise soziale Aktivitäten zum Themenfeld LSBTI*), Trainings zum Diversity Management und Biographiearbeit. Eine kompetente Praxis inklusiver Altenpflege hält auch nicht-normatives Begehren und nicht-normative Körper für möglich und erkennt spezifische Bedarfe sowie Ressourcen und Strategien. Der unerlässliche Blick auf Biografien der zu Pflegenden und die aus der Lebensgeschichte sich erklärenden Vulnerabilitäten erfordern auch die Profession der Sozialen Arbeit in der Altenpflege und damit eine verbesserte interprofessionelle Zusammenarbeit von Pflege und Sozialer Arbeit - nicht nur für LSBTI*Senior*innen, sondern für eine gute Pflegequalität im Alter für Alle.

Die Literaturliste finden Sie im HEILBERUFE eMag auf springerpflege.de

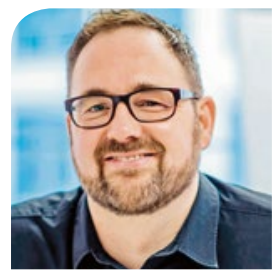

\section{Dr. Ralf Lottmann}

Wissenschaftlicher Mitarbeiter Alice Salomon Hochschule Berlin und Kuratorium Deutsche Altershilfe lottmann@ash-berlin.eu

\section{Hier steht eine Anzeige.}

\section{Springer}

